Note

\title{
ON THE ERDŐS-GYÁRFÁS CONJECTURE IN CLAW-FREE GRAPHS
}

\author{
Pouria Salehi Nowbandegani ${ }^{1}$, Hossein Esfandiari ${ }^{2}$ \\ Mohammad Hassan Shirdareh Haghighi ${ }^{1}$ and Khodakhast BibaK ${ }^{3}$ \\ ${ }^{1}$ Department of Mathematics \\ Shiraz University \\ Shiraz 71454, Iran \\ ${ }^{2}$ Department of Computer Science \\ University of Maryland College Park \\ College Park, MD 20742, USA \\ ${ }^{3}$ Department of Combinatorics and Optimization \\ University of Waterloo \\ Waterloo, Ontario, Canada N2L 3G1 \\ e-mail: pouria.salehi@gmail.com \\ hossein@cs.umd.edu \\ shirdareh@susc.ac.ir \\ kbibak@uwaterloo.ca
}

\begin{abstract}
The Erdős-Gyárfás conjecture states that every graph with minimum degree at least three has a cycle whose length is a power of 2 . Since this conjecture has proven to be far from reach, Hobbs asked if the Erdős-Gyárfás conjecture holds in claw-free graphs. In this paper, we obtain some results on this question, in particular for cubic claw-free graphs.
\end{abstract}

Keywords: Erdős-Gyárfás conjecture, claw-free graphs, cycles.

2010 Mathematics Subject Classification: C5038, C5038.

\section{REFERENCES}

[1] J.A. Bondy, Extremal problems of Paul Erdős on circuits in graphs, in: Paul Erdős and his Mathematics, II, Bolyai Soc. Math. Stud., 11, Janos Bolyai Math. Soc., Budapest (2002), 135-156.

[2] J.A. Bondy and U.S.R. Murty, Graph Theory (Springer-Verlag, New York, 2008). 
[3] D. Daniel and S.E. Shauger, A result on the Erdös-Gyárfás conjecture in planar graphs, Congr. Numer. 153 (2001) 129-140.

[4] P. Erdős, Some old and new problems in various branches of combinatorics, Discrete Math. 165/166 (1997) 227-231. doi:10.1016/S0012-365X(96)00173-2

[5] K. Markström, Extremal graphs for some problems on cycles in graphs, Congr. Numer. 171 (2004) 179-192.

[6] P. Salehi Nowbandegani and H. Esfandiari, An experimental result on the ErdösGyárfás conjecture in bipartite graphs, 14th Workshop on Graph Theory CID, September 18-23, 2011, Szklarska Poręba, Poland.

[7] S.E. Shauger, Results on the Erdös-Gyárfás conjecture in $K_{1, m}$-free graphs, Congr. Numer. 134 (1998) 61-65.

[8] J. Verstraëte, Unavoidable cycle lengths in graphs, J. Graph Theory 49 (2005) 151167.

doi:10.1002/jgt.20072

Received 29 August 2012

Revised 6 February 2013

Accepted 6 February 2013 